\title{
Oncologic surgery: a great challenge.
}

\section{Cirurgia oncológica: um grande desafio.}

Luiz Antonio Santini Rodrigues da Silva, ECBC-RJ'.

A special issue of The Lancet Oncology 1 , on September 2015, presented the report of a special committee, coordinated by Richard Sullivan, from the Institute of Cancer Policy, King's Health Partners Comprehensive Cancer Centre in London, England. Entitled "Global Cancer Surgery: delivering safe, affordable and timely cancer surgery," the report included 43 experts and managers from around the world and sought to build a picture of the state of the art surgical oncology globally. The document also brings suggestions and recommendations for countries and institutions interested in the subject. Accompanied by the Brazilian physician Audrey Tsunoda, I had the opportunity to participate in this group.

The forecasts for growth in the cancer incidence and mortality in the world, presented in another recent publication, the Atlas of Cancer $^{2}$, are catastrophic. The estimate is an increase in the number of new cases from about 14 million, in 2012, to 19.3 million in 2025. As for mortality, it is projected an increase from approximately eight million deaths that occurred in 2012, to 11.4 million in 2025. One should also consider the trend that cancer is to become the leading cause of death in all regions of the world in the coming decades, regardless of socioeconomic status.

To address the serious problem of the increased incidence of cancer, many actions have been developed in the prevention field, some with enough efficiency and visibility, as the reduction of smoking, in which Brazil is a world reference. Early detection campaigns and vaccines, such as hepatitis $B$ and, more recently, HPV, are also featured. The World Health Organization estimates that effective preventive measures could reduce by $40 \%$ the incidence of new cases of cancer. Regarding these data, the committee's report draws attention to the fact that, of about 15.2 million new cancer cases occurred in 2015, $80 \%$ must have needed a surgical procedure at some point of the disease evolution. Rob Brierley and David Collingrige ${ }^{3}$ stated that despite the advances in the field of radiotherapy and chemotherapy, surgery remains the cornerstone of cancer care, playing many roles in the prevention, diagnosis, curative treatment, supportive measures, palliation treatment and reconstructions. In this sense, the authors consider surgery a vital specialty for reducing premature mortality due to cancer.

The report concluded that the overall picture shows great disparity and inequity in access to surgery and to economic resources. Most patients do not have access to cancer surgery. Failures in forming and training of more surgeons and the weakening of health systems can result in a cumulative loss of about 6.2 trillion dollars of the global gross domestic product by the year 2030 . Supply and quality problems of surgery essential support services, such as pathology, imaging and anesthesia were also mentioned in the document. The lack of investment in access to organized services, research, training and education is strongly demonstrated, especially in countries of low and medium development. The report recognizes that there are some innovations and solutions that need to be known and utilized as examples of efforts being carried out, including Brazil.

Among the pros, I highlight the existence of the Unified Health System (SUS) in Brazil. A national system with universal coverage, regulated by a specific legislation and ministerial decrees to establish the amplitude of services to be offered according to technical training and technological resources of providers. It is also worth noting that the incorporation of technologies or new procedures are regulated by the Comissão Nacional de Incorporação de Tecnologias no SUS (CONITEC - National

1 - Departamento de Cirurgia Geral e Especializada da Universidade Federal Fluminense (UFF), Niterói, RJ, Brasil. 
Commission for Incorporation of Technologies into SUS).

There is also a permanent council formed by scientific organizations, managers and other representative institutions and coordinated by the Instituto Nacional do Câncer José Alencar Gomes da Silva (INCA). The INCA Advisory Council (CONSINCA) prepares and submits technical studies and reports to the Ministry of Health.

A a negative note, there is the imbalance of funding between the different forms of cancer treatment. Only $9 \%$ of the total funds allocated to Oncology are assigned to oncologic surgery. In almost all countries there is a growing imbalance in the sources of funds for the public sector, with most of the resources flowing through and to the private sector, which increases inequality. Of the resources for research in the world, only $9 \%$ are intended for surgery, and a small fraction of this percentage is intended for clinical trials, which are effectively able to promote the best outcomes for patients. Although cancer control can not do without cooperation between surgery, radiotherapy and chemotherapy, I draw attention to the results obtained with surgery, which can be much more positive, strongly depending on high-quality training of surgeons.

Hence I believe that cooperation between the Colégio Brasileiro de Cirurgiões ( $\mathrm{CBC}$ ) and the Sociedade Brasileira de Cirurgia Oncológica (SBCO) could have a huge meaning to meet this challenge. The starting point? Cooperation for the development of an action plan based on data from the aforementioned report that is being deepened with Brazil's data by SBCO.

\section{REFERENCES}

1. Sullivan R, Alatise Ol, Anderson BO, Audisio R, Autier $P$, Aggarwal A, et al. Global câncer surgery: delivering safe, affordable, and timely câncer surgery. Lancet Oncol. 2015;16(11):1193-224.

2. American Cancer Society [homepage on Internet]. The Cancer Atlas. 2nd ed. Available from: http://canceratlas.cancer.org/

3. Brierley R, Collingridge D. Cancer surgery: a vital speciality to prevent premature death. Lancet Oncol. 2015;16(11):1187. 